\title{
AUTOESTIMA Y AUTOEFICACIA DE LOS ALUMNOS DE EDUCACIÓN PRIMARIA EN LA ASIGNATURA DE CIENCIAS DE LA NATURALEZA
}

\author{
Irene Del Rosal \\ Dpto. de Didáctica de las Ciencias Experimentales y de las Matemáticas. Facultad de Educación, Badajoz. \\ Universidad de Extremadura, España \\ irenedelrosal@gmail.com \\ Maria Luisa Bermejo \\ Dpto. Psicología y Antropología. Facultad de Educación, Badajoz. Universidad de Extremadura, España \\ mbermejo@unex.es
}

Fecha de Recepción: 24 Febrero 2018

Fecha de Admisión: 10 Abril 2018

\section{RESUMEN}

En el ámbito de las ciencias, el interés de los estudiantes comienza pronto, pero se vuelve decreciente en la etapa de secundaria, considerando la Ciencia como algo aburrida y poco útil. La presente investigación, englobada dentro del proyecto del Plan Nacional (EDU2016-77007-R), está centrada en la Educación Primaria, momento en el cual comienzan a cristalizar las emociones de los estudiantes. Los objetivos de la presente investigación son conocer el nivel de autoestima y autoeficacia del alumnado de Educación Primaria, además de intentar encontrar diferencias en cuanto a variables sociodemográficas como el sexo y el curso de los estudiantes. La muestra de nuestro estudio está constituida por 151 alumnos de $4^{\circ}, 5^{\circ}$ y 6ํํㅁ de diferentes colegios públicos de Extremadura y el proceso de muestreo que se ha llevado a cabo para seleccionar a los sujetos encuestados ha consistido en un muestreo no probabilístico de conveniencia o incidental. Para llevar a cabo nuestra investigación hemos optado por un diseño no experimental, puesto que no hemos contado con el control de una variable independiente, ni hemos llevado a cabo ninguna intervención o tratamiento. Además, se trata de un estudio transversal o transeccional, puesto que se analiza el nivel y/o estado de diversas variables en un punto concreto del tiempo. Entre los resultados más relevantes destacan las diferencias estadísticamente significativas en cuanto al curso en el que se encuentran matriculados los estudiantes.

Palabras claves: autoestima; autoeficacia; educación primaria; ciencias de la naturaleza

\section{ABSTRACT \\ Self-esteem and self-efficacy of primary education school students in the natural sciences subject.}


In the field of science, the interest of students begins soon, but it becomes decreasing in the secondary stage, considering Science as something boring and unhelpful. The present research, included in the National Plan project (EDU2016-77007-R), is focused on Primary Education, at which point the emotions of the students begin to crystallize. The objectives of this research are to know the level of self-esteem and self-efficacy of Primary Education students, in addition to trying to find differences in sociodemographic variables such as sex and the course of students. The sample of our study is constituted by 151 students of 4th, 5th and 6th grade from different public schools of Extremadura and the sampling process that has been carried out to select the subjects surveyed has consisted in a non-probabilistic sampling of convenience or incidental. To carry out our research we opted for a non-experimental design, since we have not had control of an

independent variable, nor have we carried out any intervention or treatment. In addition, it is a cross-sectional or transectional study, since the level and / or state of various variables are analyzed at a specific point in time. Among the most relevant results stand out the statistically significant differences in the course in which the students are enrolled.

Keywords: self esteem; self-efficacy; primary education; Natural Sciences

\section{ANTECEDENTES}

De acuerdo con Borrachero (2015), creencia y emoción son dos conceptos que están muy relacionados y que dependen uno del otro. Las creencias motivacionales condicionan las características emocionales del sujeto. De esta forma, proporcionan información acerca de los sentimientos y emociones de una persona y sirven de base para posteriores decisiones, comportamientos y acciones.

El concepto "autoestima", dentro del ámbito de la psicología, es uno de los más complejos y difíciles de definir, ya que en los últimos años ha sido delimitado de forma imprecisa y confusa e incluso se ha utilizado en todo tipo de campos. A pesar de ello, desde la década de los 80, comienzan a identificarse enfoques explicativos, así como investigaciones psicológicas que intentan dar explicación al comportamiento humano (González, 1999). García, Calvo y Marrero (2006) entienden que la autoestima es "el sentimiento de aceptación y aprecio hacia uno mismo, que va unido al sentimiento de competencia y valía personal” (p. 200). Así, la autoestima incluiría dos aspectos esenciales: el sentimiento de autoeficacia y el sentimiento de ser valioso; es decir, la autoestima vendría dada por la suma de la confianza y el respeto por uno mismo. En palabras de estos autores:

Desarrollar la autoestima es desarrollar la convicción de que uno es competente para vivir y merece la felicidad, y por lo tanto enfrentarse a la vida con mayor confianza y optimismo, o cual nos ayuda a alcanzar nuestras metas. Desarrollar la autoestima es ampliar nuestra capacidad de ser felices (García, et al., 2006, p.201).

Las creencias que tiene un sujeto sobre sí mismo no se basan únicamente en conclusiones independientes que el sujeto realice sobre él, sino que influyen las ideas o comentarios que formulan las personas que lo rodean. Las instituciones y agentes involucrados en el proceso de socialización juegan un papel importante en el desarrollo de la autoestima, ya sea de forma positiva o negativa (Naranjo, 2007).

Es importante también destacar que en el ámbito de la psicología, al comienzo de la segunda revolución cognitiva, se origina un nuevo paradigma contrapuesto al conductismo. En dicho paradigma se plantea el estudio de los procesos internos del individuo, identificándolos como procesos dinámicos del sujeto. Es aquí, cuando Bandura se apoya en su teoría social cognitiva, abandonando el determinismo ambiental de la teoría conductista y describiendo al ser humano como un sujeto capaz de crear y modificar sus propias condiciones de vida. Así, y tras finalizar la década de los setenta, se propone la "Teoría de la Autoeficacia" (Valencia, 2006; Velásques, 2012). 
Por tanto, al hablar de autoeficacia debemos hacer referencia a Bandura (1997), quien afirma que este término tiene un carácter no tan global como la autoestima, puesto que estaría relacionado con las propias habilidades para la organización y ejecución de aquellas acciones que son necesarias para conseguir ciertos logros o resultados. Así, la autoeficacia se definirá como "las creencias en la propia capacidad para organizar y ejecutar las acciones requeridas para manejar las situaciones futuras" (Bandura, 1997, p. 2).

En el ámbito de las ciencias, Brígido y Borrachero (2011) mantienen que existe una relación importante entre el autoconcepto, la autoeficacia y la autorregulación en los futuros maestros de ciencias. Así, quienes poseen un alto nivel de autoconcepto, muestran altos niveles de autoeficacia y creencias de autorregulación positivas; igualmente, los maestros en formación con un alto nivel de autoeficacia en el proceso docente presentarán creencias positivas de autoeficacia. Además, desarrollar este tipo de competencia sería esencial para la formación inicial de los maestros, puesto que serán referentes para el alumnado e influirán de forma directa 0 indirecta en el comportamiento de éstos (Borrachero, Brígido, Gómez y Bermejo, 2012). En palabras de Brígido y Borrachero (2011), los futuros maestros:

Podrán tomar conciencia de que pueden ser vulnerables emocionalmente, de su propia historia como escolares y de cómo las emociones afectan a la enseñanza de las distintas asignaturas de ciencias. Además podrán desarrollar la capacidad de actuar para transformar y autorregular esas emociones, en su propio aprendizaje, en el de sus alumnos y en su enseñanza de las ciencias, en un proceso metacognitivo (p.226).

\section{OBJETIVOS DE LA INVESTIGACIÓN}

La presente investigación, englobada dentro del proyecto del Plan Nacional (EDU2016-77007R), está centrada en la etapa de Educación Primaria, momento en el cual comienzan a cristalizar las emociones de los estudiantes hacia las ciencias de la naturaleza. Los objetivos de la presente investigación son los siguientes:

Conocer el nivel de autoestima de los alumnos de educación primaria en la asignatura de Ciencias de la Naturaleza.

Conocer el nivel de autoeficacia de los alumnos de educación primaria en la asignatura de Ciencias de la Naturaleza.

Encontrar diferencias en cuanto al sexo y al curso de los alumnos de educación primaria en la asignatura de Ciencias de la Naturaleza.

\section{PARTICIPANTES}

El proceso de muestreo que se ha llevado a cabo para seleccionar a los sujetos encuestados ha consistido en un muestreo no probabilístico de conveniencia o incidental. La muestra está constituida por 151 alumnos de Educación Primaria de diferentes colegios públicos de la comunidad autónoma de Extremadura.

Si nos centramos en el curso, el $25.8 \%$ son alumnos de $4^{0}$ curso; el $44.4 \%$ son alumnos de $5^{\circ}$ curso y el $29.8 \%$ restantes, son los alumnos de 6을 curs. Si nos centramos en el sexo, el $58.3 \%$ son niños, mientras que el $41.7 \%$ restante son niñas.

\section{METODOLOGÍA E INSTRUMENTOS}

Para llevar a cabo nuestra investigación hemos optado por un diseño no experimental, puesto que no hemos contado con el control de una variable independiente, ni hemos llevado a cabo ninguna intervención o tratamiento. Además, se trata de un estudio transversal 0 transeccional, puesto que se analiza el nivel y/o estado de diversas variables en un punto concreto del tiempo. 
Este estudio surge por la necesidad de validar un cuestionario de elaboración propia que nos permita conocer qué emociones experimentan los alumnos de Educación Primaria en la asignatura de Ciencias de la Naturaleza ante ciertos contenidos.

Para analizar la autoestima y autoeficacia del alumnado de Educación Primaria se han seleccionado y adaptado ítems de distintas escalas (Baessler, J. y Schwarcer, R., 1996; Bandura, 1997; Mérida, R., Serrano, A., Tabernero, C., 2015; Palenzuela, 1983; Rosenberg, M., 1965; Sanjuán, P., Pérez, A.M. y Bermúdez, J., 2000). En la Tabla 1 se recogen los distintos ítems que conforman nuestra escala tipo Likert de 4 puntos según la dimensión evaluada dentro de la asignatura de Ciencias de la Naturaleza.

Tabla 1: Ítems para la evaluación de la autoeficacia y autoestima.

\begin{tabular}{lll}
\hline & \multicolumn{1}{c}{ ÍTEMS } \\
\hline \multirow{3}{*}{ Autoeficacia } & - & Soy capaz de realizar las actividades. \\
& - & Si me lo propongo, creo que soy capaz de sacar buenas \\
& notas. \\
& - & Soy capaz de comprender bien los temas. \\
& - & Puedo resolver la mayoría de las tareas si me esfuerzo. \\
\hline Autoestima & - & Pienso que la asignatura de CCNN se me da bastante bien. \\
& - & El maestro dice que trabajo bien en clase. \\
& - & Soy bueno en la asignatura de CCNN. \\
\hline
\end{tabular}

\section{RESULTADOS}

A continuación se muestran los resultados de nuestro estudio relacionados el nivel de autoestima y autoeficacia de los alumnos de $4^{\circ}, 5^{\circ}$ y $6^{\circ}$ de Educación Primaria.

En la Figura 1 podemos identificar el nivel de autoestima de los participantes de nuestra investigación según el sexo. Como podemos observar son las niñas quienes obtienen mayores puntuaciones, sin embargo, y tras aplicar las pruebas correspondientes, estas diferencias no son estadísticamente significativas.

Figura 1. Autoestima según el sexo

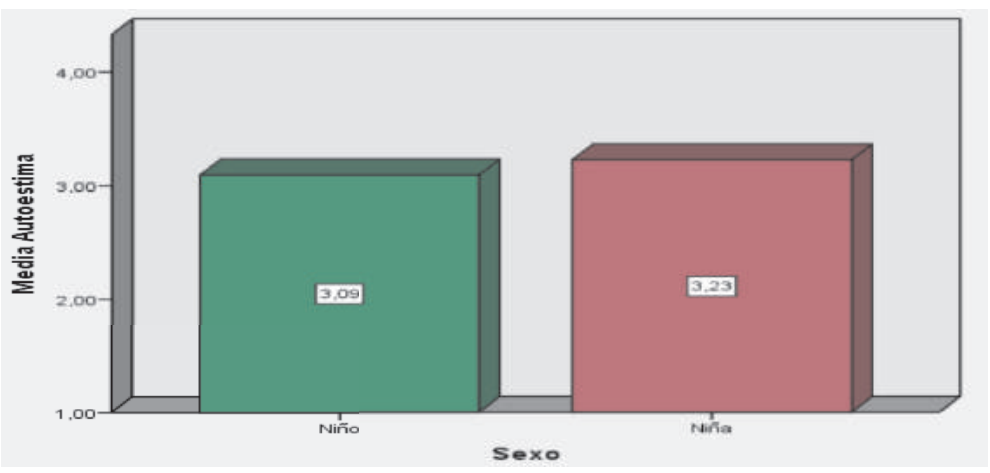


En la Figura 2 se muestra el nivel de autoeficacia de los participantes de nuestra investigación según el sexo. En esta ocasión, son los niños quienes obtienen mayores puntuaciones, sin embargo estas diferencias no son estadísticamente significativas.

Figura 2. Autoeficacia según el sexo

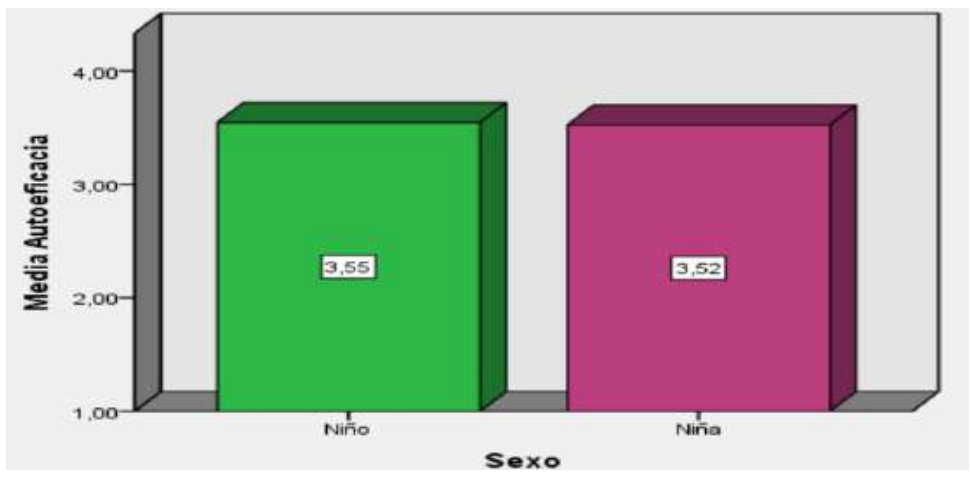

Si nos centramos ahora en el nivel a autoestima según el curso en el que se encuentran matriculados los participantes de nuestro estudio, y tal y como podemos observar en la Figura 3, los alumnos de $4^{0}$ curso presentan mayores niveles de autoestima que los alumnos de $5^{\circ}$ y $6^{0}$ curso de Educación Primaria. Tras aplicar las pruebas estadísticas correspondientes, podemos afirmar que estas diferencias según el curso sí son estadísticamente significativas.

Figura 3. Autoestima según el curso

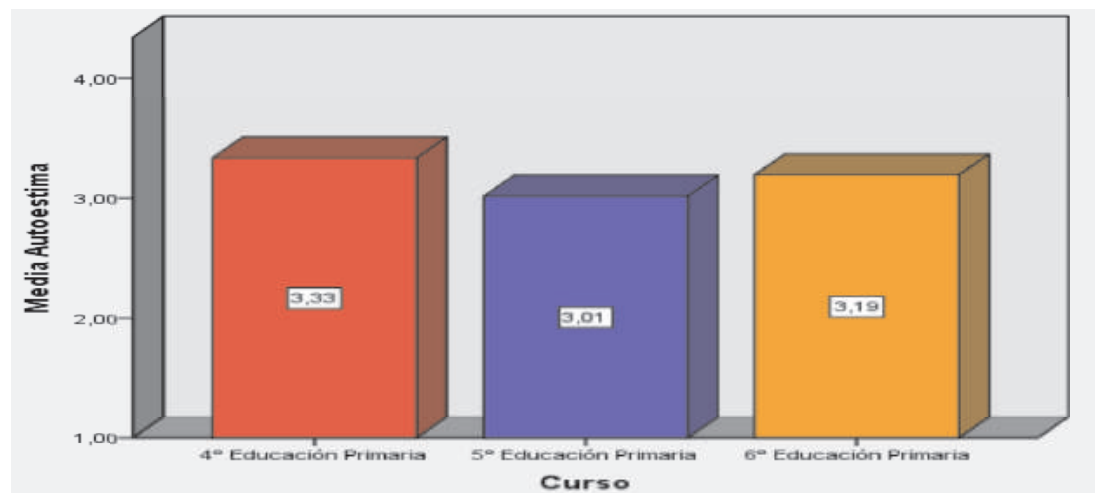

Por último, en la Figura 4, se muestra el nivel de autoeficacia de los alumnos participantes en nuestra investigación según el curso. De nuevo, son los alumnos de $4^{0}$ curso quienes obtienes mayores puntuaciones en el nivel de autoeficacia, siendo estas diferencias estadísticamente significativas. 
Figura 4. Autoeficacia según el curso

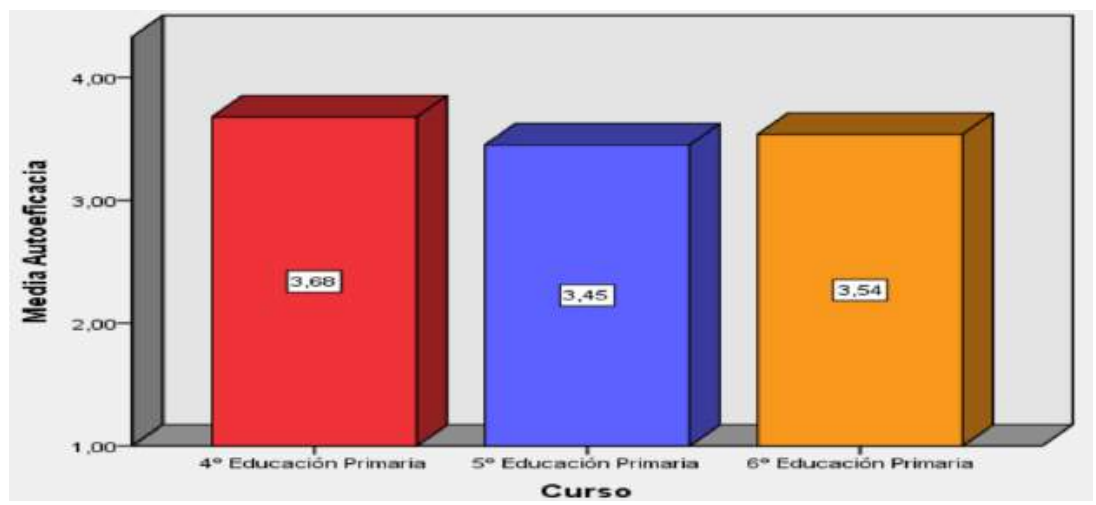

\section{DISCUSIÓN Y CONCLUSIONES}

Con el presente estudio hemos analizado el nivel de autoestima y autoeficacia de los alumnos de Educación Primaria ante la asignatura de Ciencias de la Naturaleza según variables sociodemográficas como el sexo y el curso, encontrándose diferencias estadísticamente significativas en esta última variable.

Nuestros resultados van en la línea de Carrasco y del Barrio (2002) quienes defienden que el nivel de autoeficacia disminuye con los grupos de mayor edad. Por ello, los sujetos con menor edad muestran mayores niveles de autoeficacia académica, social y de control. La llegada de la adolescencia, tanto en chicos como en chicas, conlleva una progresiva disminución de la autoeficacia; en esta misma línea, Bandura (1997) señala que la adolescencia es un periodo de transición y turbulencia, donde cabe esperar un descenso en el nivel de autoeficacia

Como se refleja en numerosos estudios existe una relación significativa entre el autoconcepto, la autoeficacia y la autorregulación de los sujetos. Por tanto, fomentar este tipo de competencias sería un aspecto esencial para la formación inicial de los maestros, puesto que serán referentes para el alumnado e influirán de forma directa o indirecta en el comportamiento de éstos (Brígido y Borrachero, 2011; Borrachero et al., 2012; Del Rosal y Bermejo, 2017).

\section{REFERENCIAS BIBLIOGRÁFICAS}

Baessler, J. y Schwarcer, R. (1996). Evaluación de la autoeficacia: Adaptación española de la escala de Autoeficacia General. Ansiedad y Estrés, 2, 1-8.

Bandura, A. (1997). Self-Efficacy. The exercise of control. New Jersey: Freeman.

Borrachero, A.B. (2015). Las Emociones en la Enseñanza y el Aprendizaje de las Ciencias en Educación Secundaria. Tesis Doctoral (Inédita). Universidad de Extremadura: Badajoz.

Borrachero, A.B., Brígido, M., Gómez, R. y Bermejo, M.L. (2012). Relación entre autoconcepto y autoeficacia en los futuros profesores de secundaria. International Journal of Developmental and Educational Psychology: INFAD. Revista de Psicología, 1(2), 219-226

Borrachero, A. B., Brígido, M., Gómez, R. y Bermejo, M.L. (2012). Relación entre autoconcepto y autoeficacia en los futuros profesores de Secundaria. International Journal of Developmental and Educational Psychology, 2(1), 21-226.

Brígido, M. y Borrachero, A.B. (2011). Relación entre autoconcepto, autoeficacia y autorregulación en ciencias de futuros maestros de Primaria. International Journal of Developmental and 
Educational Psychology: INFAD. Revista de Psicología, 1(2), 107-113.

Carrasco, M.A. y Del Barrio, M.V. (2002). Evaluación de la autoeficacia en niños y adolescentes. Psicothema, 14(2), 323-332.

Del Rosal, I. y Bermejo, M.L. (2017). Autoeficacia en estudiantes universitarios: diferencias entre el grado de maestro en educación primaria y los grados en ciencias. International Journal of Developmental and Educational Psychology, 1(1), 115-123.

García, A., Calvo, P. y Marrero, G. (2006). La autoestima en alumnos de sexto curso de educación primaria. En F. Bacaicoa y J. Uriarte (Eds.). Psicología del Aprendizaje, INFAD, 199-215. Universidad de Extremadura: Psicoex Badajoz.

González, M.T. (1999). Algo sobre la autoestima. Qué es y cómo se expresa. Aula, 11, 217-232.

Mérida, R., Serrano, A. y Tabernero, C. (2015). Diseño y validación de un cuestionario para la evaluación de la autoestima en la infancia. Revista de Investigación Educativa, 33(1), 149-162.

Naranjo, M.L. (2007). Autoestima: un factor relevante en la vida de la persona y tema esencial del proceso educativo. Actualidades investigativas en Educación, 7(3), 1-27.

Palenzuela, D. (1983). Construcción y validación de una escala de autoeficacia percibida específica de situaciones académicas. Análisis y Modificación de Conducta, 9(21), 185-219.

Rosenberg, M. (1965). Society and the adolescent self-image. Princeton, NJ: Princeton University Press.

Sanjuán, P., Pérez A.P. y Bermúdez, J. (2000). Escala de autoeficacia general: datos psicométricos de la adaptación para población española. Psicothema, 12(Suplemento), 509-513.

Valencia, L. (2006). Teoría del aprendizaje social y/o teoría cognitivo social de Bandura. En L. Bardales, P. Díaz, M. Jiménez, M. Terreros y L. Valencia, (Eds.) Psicología Social: Pasado, Presente y Futuro, 193-221. Universidad del Valle: Instituto de Psicología.

Velásques, A. (2012). Revisión histórico-conceptual del concepto de autoeficacia. Pequén, 1(2), 148-160. 
